\title{
INVESTIGATIVE UROLOGY
}

\section{Comparison of gene expression profiles between Peyronie's disease and Dupuytren's contracture \\ Qian A, Meals RA, Rajfer J, Gonzalez-Cadavid NF}

Harbor-UCLA Research and Education Institute, Torrance, California, Department of Orthopedics and Urology, University of California, Los Angeles, School of Medicine, Los Angeles, California, USA Urology 2004; 64: 399-404

Objectives: To compare the gene expression alterations in human Peyronie's disease (PD) and Dupuytren's disease (DD) to determine whether they share a common pathophysiology. Multiple mRNA expression profiles of human PD have previously shown that genes that regulate fibroblast replication, myofibroblast differentiation, collagen metabolism, tissue repair, and ossification are involved. DD, a palmar fascia fibrosis, may be associated with PD.

Methods: Total RNA samples from PD plaques, normal tunica albuginea, Dupuytren's nodules, and normal palmar fascia (nine samples per group) were subjected to differential gene expression profile analysis (Clontech Atlas DNA microarray) comparing PD with tunica albuginea and DD with normal palmar fascia. Changes of more than 2.0 in PD and DD compared with tunica albuginea and normal palmar fascia, respectively, were recorded. Reverse transcriptase-polymerase chain reactions were performed for some genes whose expression was altered in PD.

Results: Some of the gene families upregulated in both PD and DD were (a) collagen degradation: matrix metalloproteinase (MMP), with MMP2 and MMP9, and thymosins (MMP activators), with TM $\beta 10$ and TM $\beta 4$; (b) ossification: osteoblast-specific factors (OSFs) OSF-1 and OSF-2 (DD only); and (c) myofibroblast differentiation: RhoGDP dissociation inhibitor 1. The genes upregulated in PD only were decorin (an inhibitor of transforming growth factor-beta1 and a part of fibroblast replication/collagen synthesis) and early growth response protein. Reverse transcriptase-polymerase chain reaction confirmed these changes.

Conclusions: These data demonstrate that the pattern of alterations in the expression of certain gene families in PD and DD is similar, suggesting that they share a common pathophysiology and may be amenable to the same therapeutic regimens.

\section{Editorial Comment}

The authors present one more wonderful contribution to the knowledge of Peyronie's disease.

One of the most accepted etiologies for Peyronie's disease is that it is caused by trauma to the erect penis, resulting in extravasation of fibrin and other blood proteins into the tunica albuginea that, together with other unknown factors, elicit an inflammatory reaction followed by the production of pro-fibrotic agents, such as transforming growth factor-beta1 and reactive oxygen species. Peyronie's disease may be associated with Dupuytren's disease, which occurs in the palmar fascia in $21 \%$ of the cases. Dupuytren's disease is characterized by similar fibrotic alterations, although its relationship to trauma is less established.

Analyzing gene expression, this study provides targets of potential pharmacologic modulation of the levels of genes associated with antifibrotic mechanisms. The authors speculate that stimulation of myofibroblast apoptosis and blockade of its differentiation with Rho inhibitors or cortactin may be beneficial, because accumulation of these cells in an abnormal healing process subsequent to trauma may relate to the fibrosis seen in Peyronie's disease and Dupuytren's disease.

Previous studies by the same group (1) demonstrated that treatment with L-arginine and phosphodiesterase inhibitors, both stimulating apoptosis and remodeling by nitric oxide/cyclic guanosine monophosphate or cyclic guanosine monophosphate alone, respectively, has been shown to prevent the fibrotic plaque in the TGF- $\beta$ animal model of Peyronie's disease. 


\title{
REFERENCE
}

1. Valente EG, Vernet D, Ferrini MG, Qian A, Rajfer J, Gonzalez-Cadavid NF: L-arginine and phosphodiesterase (PDE) inhibitors counteract fibrosis in the Peyronie's fibrotic plaque and related fibroblast cultures. Nitric Oxide. 2003; 9 : $229-44$.

\author{
Dr. Francisco J.B. Sampaio \\ Full-Professor and Chair, Urogenital Research Unit \\ State University of Rio de Janeiro \\ Rio de Janeiro, Brazil
}

\section{Effects of peppermint teas on plasma testosterone, follicle-stimulating hormone, and luteinizing hormone levels and testicular tissue in rats \\ Akdogan M, Ozguner M, Kocak A, Oncu M, Cicek E \\ Department of Biochemistry, Department of Histology-Enbriology, Department of Pharmacology, Suleyman \\ Demirel University Medical School, Isparta, Turkey \\ Urology 2004; 64: 394-8}

Objectives: To justify the effects of Mentha piperita labiatae and Mentha spicata labiatae herbal teas on plasma total testosterone, luteinizing hormone, and follicle-stimulating hormone levels and testicular histologic features. We performed this study because of major complaints in our area from men about the adverse effects of these herbs on male reproductive function.

Methods: The experimental study included 48 male Wistar albino rats (body weight 200 to $250 \mathrm{~g}$ ). The rats were randomized into four groups of 12 rats each. The control group was given commercial drinking water, and the experimental groups were given $20 \mathrm{~g} / \mathrm{L}$ M. piperita tea, $20 \mathrm{~g} / \mathrm{L}$ M. spicata tea, or $40 \mathrm{~g} / \mathrm{L} \mathrm{M}$. spicata tea.

Results: The follicle-stimulating hormone and luteinizing hormone levels had increased and total testosterone levels had decreased in the experimental groups compared with the control group; the differences were statistically significant. Also, the Johnsen testicular biopsy scores were significantly different statistically between the experimental groups and the control group. Although the mean seminiferous tubular diameter of the experimental groups was relatively greater than in the control group, the difference was not statistically significant. The only effects of M. piperita on testicular tissue was segmental maturation arrest in the seminiferous tubules; however, the effects of M. spicata extended from maturation arrest to diffuse germ cell aplasia in relation to the dose.

Conclusions: Despite the beneficial effects of M. piperita and M. spicata in digestion, we should also be aware of the toxic effects when the herbs are not used in the recommended fashion or at the recommended dose.

\section{Editorial Comment}

This is the first report concerning the effects of peppermint tea on plasma total testosterone, $\mathrm{LH}$, and FSH levels and testicular histologic features.

Peppermint tea is generally considered a safe drink for regular consumption. The authors demonstrate that both M. piperita and M. spicata tea intake decreased plasma testosterone and increased plasma LH and FSH levels in rats. Histologic studies revealed extensive degenerative changes in the germinal epithelium and spermatogenesis arrest when compared to controls.

Changes in the pituitary-testicular axis may be responsible for the testicular maturation arrest. The statistically significant decrease in both spermatogenesis and plasma total testosterone levels in the experimen- 
tal groups was associated with an increase in the plasma FSH and LH levels. These observations prompted the authors to consider the pituitary-testicular axis. The plasma total testosterone levels had decreased and plasma FSH and LH levels increased, as expected. Therefore, the mechanism of spermatogenic abnormalities was more likely a result of the direct effect on germinal epithelium, and the hormonal deficit appeared to be a result of Leydig cell dysfunction. The pituitary gland or hypothalamus may also be affected, and the maturation arrest could have been the result of hypothalamic-pituitary-testicular axis deficiency. However, this hypothesis should be elucidated by additional studies focused on the hypophysial or hypothalamic tissues.

Consumption of M. piperita and M. spicata teas affected spermatogenetic activity at the $20 \mathrm{~g} / \mathrm{L}$ and 40 $\mathrm{g} / \mathrm{L}$ dose, respectively, in rats. The authors remember us that despite M. piperita and M. spicata beneficial effects in digestion, people should be aware of their toxic adverse effects when not used in the recommended fashion or at the recommended dose.

\author{
Dr. Francisco J.B. Sampaio \\ Full-Professor and Chair, Urogenital Research Unit \\ State University of Rio de Janeiro \\ Rio de Janeiro, Brazil
}

\title{
RECONSTRUCTIVE UROLOGY
}

\section{Current and future strategies for preventing and managing erectile dysfunction following radical prostatectomy}

Montorsi F, Briganti A, Salonia A, Rigatti P, Burnett AL

Department of Urology, Università Vita-Salute San Raffaele, Via Olgettina 60, 20132, Milan, Italy, and Department of Urology, The Johns Hopkins Hospital, Baltimore, Maryland, USA

Eur Urol. 2004; 45: 123-133

Introduction and Objectives: As radical prostatectomy remains a commonly used procedure in the treatment of clinically localized prostate cancer, we critically analyzed current and future strategies for preventing and managing postoperative erectile dysfunction.

Methods: Systematic literature review using Medline and CancerLit from January 1997 to June 2003. Abstracts published in the journals European Urology, The Journal of Urology and the International Journal of Impotence Research as official proceedings of internationally known scientific societies held in the same time period were also assessed.

Results: Patient selection and surgical technique are the major determinants of postoperative erectile function. Apoptosis of corporeal smooth muscle cells plays a role in the development of cavernous venoocclusive dysfunction following radical prostatectomy. Pharmacological prophylaxis and treatment of postoperative erectile dysfunction is effective and safe. The concepts of cavernous nerve reconstruction and neuroprotection have been associated to promising results.

Conclusions: In the hands of experienced surgeons, properly selected patients undergoing a nerve sparing radical prostatectomy should achieve unassisted or medically assisted erections postoperatively.

\section{Editorial Comment}

This paper written by a team of young experts on the treatment of sexual dysfunction nicely describes how erectile function can currently be treated after oncological pelvic surgery. It is a valuable reference for both the pelvic surgeons performing potency preserving techniques and those who deal with these patients postop- 